\title{
Calculation of the dynamic air flow resistivity of fibre materials
}

\author{
Tarnow, Viggo
}

Published in:

Acoustical Society of America. Journal

Link to article, DOI:

$10.1121 / 1.420079$

Publication date:

1997

Document Version

Publisher's PDF, also known as Version of record

Link back to DTU Orbit

Citation (APA):

Tarnow, V. (1997). Calculation of the dynamic air flow resistivity of fibre materials. Acoustical Society of America. Journal, 102(3), 1680-1688. https://doi.org/10.1121/1.420079

\section{General rights}

Copyright and moral rights for the publications made accessible in the public portal are retained by the authors and/or other copyright owners and it is a condition of accessing publications that users recognise and abide by the legal requirements associated with these rights.

- Users may download and print one copy of any publication from the public portal for the purpose of private study or research.

- You may not further distribute the material or use it for any profit-making activity or commercial gain

- You may freely distribute the URL identifying the publication in the public portal

If you believe that this document breaches copyright please contact us providing details, and we will remove access to the work immediately and investigate your claim. 


\title{
Calculation of the dynamic air flow resistivity of fiber materials
}

\section{Viggo Tarnow}

Department of Applied Engineering and Production, Technical University of Denmark, Bygning 358, DK 2800 Lyngby, Denmark

(Received 15 February 1996; revised 16 April 1997; accepted 12 May 1997)

\begin{abstract}
The acoustic attenuation of acoustic fiber materials is mainly determined by the dynamic resistivity to an oscillating air flow. The dynamic resistance is calculated for a model with geometry close to the geometry of real fiber material. The model consists of parallel cylinders placed randomly. Two cases are treated: flow perpendicular to the cylinder axes, and flow parallel to the axes. In each case two new approximate procedures were used. In the first procedure, one solves the equation of flow in a Voronoi cell around the fiber, and averages over the distribution of the Voronoi cells. The second procedure is an extension to oscillating air flow of the Brinkman self-consistent procedure for dc flow. The procedures are valid for volume concentration of cylinders less than 0.1. The calculations show that for the density of fibers of interest for acoustic fiber materials the simple self-consistent procedure gives the same results as the more complicated procedure based on average over Voronoi cells. Graphs of the dynamic resistivity versus frequency are given for fiber densities and diameters typical for acoustic fiber materials. (C) 1997 Acoustical Society of America. [S0001-4966(97)01209-5]
\end{abstract}

PACS numbers: 43.58.Bh, 43.20.Hq, 43.20.Jr, 43.55.Ev [SLE]

\section{LIST OF SYMBOLS}

A

$A_{z}$

$a$

$a_{1}, a_{2}, a_{3} \ldots$

$a_{t}$

$b$

$b_{1}, b_{2}, b_{3} \ldots$

$c_{1}, c_{2}, c_{3} \ldots$

$d_{1}, d_{2}, d_{3} \ldots$

$F_{\|}$

$F_{\perp}$

$g, h$

$i$

$k$

$k_{\|}^{\prime}$

$k_{\perp}^{\prime}$

$P$

$P(\rho)$

$p$

$\langle p\rangle$

$p_{b}(S)$

$R_{\|}$

flow

0 or 1

dius

gons vector velocity potential

$Z$ component of vector potential

radius of a cylinder

integration constants

radius of tubes in theory of porous materials

square root of area per cylinder

integration constants

integration constants

integration constants

force on a cylinder per length for parallel

force on a cylinder per length for perpendicular flow

integration constants

wave vector defined in Eq. (10)

wave vector in iterative computation for par-

allel flow

wave vector in iterative computation for per-

pendicular flow

complex power

complex power as a function of the cell ra-

local air pressure

mean pressure $=$ sound pressure

probability density of area of Voronoi poly-

resistivity for parallel flow

resistivity due to forces on cylinders for parallel flow

\section{INTRODUCTION}

We assume that sound waves in a fiber material can be described by a linear theory, and we use the complex notation for monochromatic waves with the time factor $e^{-i \omega t}$,

$R_{\perp} \quad$ resistivity for perpendicular flow

$R_{\perp}^{\prime} \quad$ resistivity due to forces on cylinders for perpendicular flow

$R(\omega) \quad$ scalar resistivity

$\mathbf{R}(\omega) \quad$ resistivity tensor

$r$

$S$

$\bar{S}$

$t$

$\mathbf{u}$

$\langle u\rangle$

$u_{r}, u_{\theta}$

$u_{z}$

V

$w$

$x, y, z$

$Z_{i}(w)$

$\eta \quad$ viscosity of air

$\theta \quad$ angle in polar coordinate system; $\tan \theta=y / x$

$\rho$

$\rho_{0} \quad$ mass density of air in equilibrium

$\rho(\omega) \quad$ effective dynamic mass density of air

$\sigma \quad$ dc resistivity

$\phi \quad$ scalar velocity potential

$\Psi \quad$ volume velocity

$\Omega \quad$ porosity Eq. (16)

$\omega \quad$ cyclic frequency

where $\omega$ is the cyclic frequency and $t$ the time. The resistivity tensor for fiber materials $\mathbf{R}(\omega)$ gives the gradient of the air pressure when the air flow velocity is given; the relation being

$-\langle\nabla p\rangle=\mathbf{R}(\omega)\langle\mathbf{u}\rangle$, 
where $\langle p\rangle$ is the mean pressure and $\langle\mathbf{u}\rangle$ the mean particle velocity vector, and mean values are taken over the volume of the fiber material. The air is regarded as an incompressible fluid in this definition of the resistivity. The resistivity will be computed from the volume concentration of the fibers and the mean diameter of the fibers, with the purpose of being able to calculate the acoustic properties such as sound velocity and attenuation in fibrous materials. These properties also depend on the compressibility, which has been calculated in Tarnow. ${ }^{1}$ In the present paper it is assumed that the fibers do not move, because it is apparently not necessary to take this into account in order to calculate the velocity and attenuation of audible sound propagation in glass wool at normal densities. $^{2}$

The standard method of describing sound propagation in fibrous materials is the theory of porous materials, which models the medium as a solid with air filled tubes of an equal radius much smaller than the wavelength of interest. The medium is regarded as isotropic. Therefore the dynamic resistivity is a scalar $R(\omega)$. Some authors use the effective mass density of air $\rho(\omega)$, which is related to the dynamic resistivity by $\rho(\omega)=-i \omega R(\omega)$. The effective mass density was computed for a circular tube by Zwikker and Kosten, ${ }^{3}$ who gave the formula

$$
\rho(\omega)=\frac{\rho_{0}}{1-2 J_{1}\left(k a_{t}\right) / k a_{t} J_{0}\left(k a_{t}\right)},
$$

where $\rho_{0}$ is the static mass density of air, $a_{t}$ is the radius of the tubes, $J_{0}(w)$ and $J_{1}(w)$ are the usual Bessel functions, and

$$
k=\sqrt{i \omega \rho_{0} / \eta},
$$

where $\eta$ is the viscosity of air.

In porous materials the effective mass density is somewhat higher than predicted by Eq. (2) because a part of the cross section is filled with solid material, which makes the air velocity in the tubes higher than the mean velocity. This depends on the density of fibers, but for acoustic fiber materials the volume density of fibers is in most cases smaller than $2 \%$, and for the sake of simplicity we therefore neglect a small increase in resistivity due to this. Therefore Eq. (2) gives the resistivity of the fiber material.

Biot $^{4}$ used the same formula written in a different way, and the author considered the coupling of waves in the solid matrix to waves in the fluid, which fills the pores. Lambert ${ }^{5}$ studied open-cell foam and used the same formula, and the author suggested ways to choose a reasonable value of the tube radius. Lambert and Tesar $^{6}$ applied the formula to fibrous material and found a way to determine the tube radius from experimental data for the static resistivity. Attenborough $^{7}$ also used Eq. (2) for one tube, but for the bulk medium $k a_{t}$ in Eq. (2) was replaced by a quantity containing the resistivity, the porosity, the tortuosity, and a pore shape factor. Stinson ${ }^{8}$ computed the dynamic mass density for rectangular tubes.

Stinson and Champoux ${ }^{9}$ showed that the pore shape factor suggested by Attenborough must depend on frequency; they also suggest a new formula for computing the dynamic mass density from the dc flow resistivity, tortuosity, porosity, and a new pore shape factor. Allard and Champoux ${ }^{10}$ gave a formula for the dynamic mass density that only requires knowledge of the dc flow resistivity.

It is the goal of the present work to calculate the dynamic resistivity of fibrous materials from the microscopic geometry of the material without introducing adjustable parameters that are used to fit predictions from models to experimental data. The purpose is to obtain a better physical understanding of the dynamic resistivity of fiber materials in order to be able to predict it when the microscopic geometry is known. The diameters of the fibers can be found by microscopy and the volume density from mass density.

We consider models that have a geometry similar to the microscopic geometry of fiber materials. In the models it is assumed that the fibers all have the same diameter, are parallel, and randomly placed. Flows parallel and perpendicular to fibers are treated.

\section{GENERAL THEORY}

The Navier-Stokes equation for incompressible stationary flow can be found in Sec. 15 of Landau and Lifschitz. ${ }^{11}$ We assume the air velocity is small-low Reynold's number. In this case the equation for the velocity vector is

$$
\rho_{0} \frac{\partial \mathbf{u}}{\partial t}=-\nabla p+\eta \nabla^{2} \mathbf{u},
$$

where $\mathbf{u}$ is the air particle velocity vector, $p$ is the local air pressure, and $t$ the time. In Pierce ${ }^{12}$ the viscosity of air is given, $\eta=1.846 \times 10^{-5} \mathrm{~kg} /(\mathrm{ms})$ at the temperature $27^{\circ} \mathrm{C}$, its temperature dependence can also be found in Pierce. ${ }^{12}$

The resistivity will be used for computing the acoustic properties of fiber materials such as glass wool. A continuum model is used to describe sound propagation in the fiber materials. The only material parameters are compressibility and resistivity. The compressibility has been computed in Tarnow. ${ }^{1}$ In a propagating sound wave at a fixed time there are places where the compression is zero. In such places one needs the resistivity of incompressible flow in order to compute the energy loss and the kinetic energy. Therefore, the assumption of only one resistivity requires that it is calculated for incompressible flow. Thus one must neglect the compressibility of air when one calculates the resistivity.

The incompressibility of the flow is expressed by

$$
\boldsymbol{\nabla} \cdot \mathbf{u}=0 .
$$

The boundary conditions for Eqs. (4) and (5) are that the velocity on the cylinders walls equals zero. The procedure for computing the resistivity is to solve these equations when 


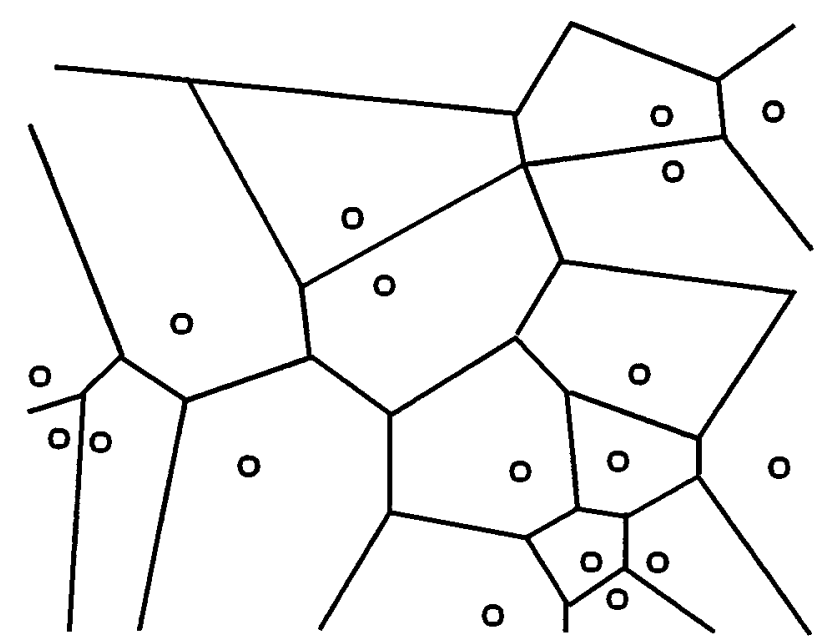

FIG. 1. The Voronoi partition of the plane. The points shown as circles are placed randomly over the plane. The lines are constructed by drawing lines from each point to the nearest neighbor point and drawing lines that bisects the lines between points. Only the bisecting lines are shown.

the mean gradient of the air pressure is given, where the mean is taken over the volume of the fiber material. From the velocity calculated from these equations one computes the mean velocity and finds the resistivity from Eq. (1).

We assume the cylinders are randomly placed. There seems to not be experimental information about the distribution of the fibers in real materials. One could assume that the fibers were placed in a regular lattice, but this is certainly not the case in glass wool. Measurements to be reported later show that for a given density the resistivity calculated for the regular lattice is too high. The resistivity for dc. flow in a regular lattice was calculated in Tarnow. ${ }^{13}$ Random placement seems to be the best model, and it can explain the measurement of sound velocity and attenuation that will be reported later.

A cell method is used to find the microscopic velocity. We take the parallel cylinders as given and cut them with a perpendicular plane; in this plane each of the centers of the cylinders is surrounded by a so-called Voronoi polygon. It is constructed by drawing lines from each center to the nearest neighbor centers and drawing lines that bisects the lines between centers. These lines form the Voronoi polygons (Fig. $1)$. The area $S$ of the Voronoi polygons has a probability $p_{b}(S) d S$ in the interval $S, S+d S$

$$
p_{b}(S)=\frac{\alpha^{\alpha}}{\Gamma(\alpha)}\left(\frac{S}{\bar{S}}\right)^{\alpha-1} \exp \left(-\alpha \frac{S}{\bar{S}}\right) \frac{1}{\bar{S}}
$$

where $\alpha=3.61, \Gamma(\alpha)$ is the gamma function, and $\bar{S}$ is the mean value of the area of the Voronoi polygons. The formula was given by Andrade and Fortes. ${ }^{14}$

The procedure is the same in each case; the NavierStokes equation is solved approximately in a Voronoi polygon, and the flow through the polygon is computed. The average flow is computed by using Eq. (6). From the average flow in a polygon the average velocity is calculated, and the resistivity is found from this. This procedure has in the case of dc flow been compared with other methods of computing the resistivity, and it was found that it yielded reliable results. ${ }^{13}$

In order to check the accuracy of the cell approximations, the resistivity was also computed by a self-consistent procedure, which is similar to the calculation of the dc resistivity by Brinkman's approximation. ${ }^{15}$

\section{FLOW PARALLEL TO CYLINDERS}

The flow is assumed to be parallel to the cylinders; in this case the gradient of the pressure does not depend on the space point, ${ }^{11}$ and the Navier-Stokes equation is easier to solve than for flow perpendicular to the cylinders. The air velocity is everywhere in the direction of the flow (see Ref. 11 ), and we assume it to be along the $Z$ axis of a rectangular $X-Y-Z$ coordinate system. The Navier-Stokes equation becomes

$$
-i \omega \rho_{0} u_{z}=-\frac{\partial p}{\partial z}+\eta\left(\frac{\partial^{2} u_{z}}{\partial x^{2}}+\frac{\partial^{2} u_{z}}{\partial y^{2}}\right) .
$$

The cylinders are parallel and randomly placed with constant density over a plane perpendicular to the cylinders, and the velocity must be zero at their surface. In this case the incompressibility condition Eq. (5) is always fulfilled.

\section{A. The cell approximation}

The Navier-Stokes equation is first solved with only one cylinder whose axis is on the $Z$ axis of the coordinate system. We use polar coordinates with radius $r$ and angle $\theta$. The boundary conditions are: $u_{z}(a)=0$, where $a$ is the radius of the cylinder, and $\partial u_{z} / \partial r=0$ on a circle $r=\rho$. The polygons are approximated by circles with different $\rho$ for each Voronoi polygon. We imagine that the velocity function is plotted above the plane of Fig. 1. Because the velocity is zero on the cylinders, the plot has a ridge close to the perimeter of each Voronoi polygon in Fig. 1, and the velocity gradient perpendicular to the sides of the polygon is close to zero. When the polygons are replaced by circles with radius $\rho$, it is reasonable to assume that the gradient along the radius is zero. In the case of dc flow this was used in Tarnow, ${ }^{13}$ where it gave accurate results.

When we use the usual complex notation for harmonic variations, the solution to the Navier-Stokes equation (7) in polar coordinates is

$u_{z}(r)=\frac{1}{i \omega \rho_{0}} \frac{\partial p}{\partial z}\left[1+\frac{J_{1}(k \rho)}{Z_{0}(k a)} H_{0}^{1}(k r)-\frac{H_{1}^{1}(k \rho)}{Z_{0}(k a)} J_{0}(k r)\right]$,

with the usual Bessel functions, and we have defined a special Bessel function $Z_{i}(w)$ of the complex variable $w$,

$$
Z_{i}(w)=H_{1}^{1}(k \rho) J_{i}(w)-J_{1}(k \rho) H_{i}^{1}(w)
$$

with

$$
k=\sqrt{\frac{i \omega \rho_{0}}{\eta}} .
$$

The function in Eq. (9) will used later. The volume velocity through a cell of a radius $\rho$ is 


$$
\Psi(\rho)=\int_{a}^{\rho} u_{z}(r) 2 \pi r d r
$$

After integration the flux becomes

$$
\begin{aligned}
\Psi(\rho)= & \frac{1}{i \omega \rho_{0}} \frac{\partial p}{\partial z}\left[\pi \rho^{2}-\pi a^{2}\right. \\
& \left.-\frac{2 \pi a\left(J_{1}(k \rho) H_{1}^{1}(k a)-H_{1}^{1}(k \rho) J_{1}(k a)\right)}{k Z_{0}(k a)}\right] .
\end{aligned}
$$

The resistivity can be found by calculating the mean velocity from Eq. (12) and using the definition of resistance Eq. (1). But it is more informative and accurate to compute the resistivity in the following way. Equation (7) is transformed by use of the Gauss integral theorem over the area of the cell; thus

$$
-i \omega \rho_{0} \Psi(\rho)=-\left(\pi \rho^{2}-\pi a^{2}\right) \frac{\partial p}{\partial z}-\eta 2 \pi a\left[\frac{\partial u_{z}}{\partial r}\right]_{r=a} .
$$

The average over the cells is computed, and one gets

$$
-i \omega \rho_{0}\langle\Psi\rangle=-\left(\pi\left\langle\rho^{2}\right\rangle-\pi a^{2}\right) \frac{\partial p}{\partial z}-\eta 2 \pi a\left\langle\left[\frac{\partial u_{z}}{\partial r}\right]_{r=a}\right\rangle,
$$

where \langle\rangle means average.

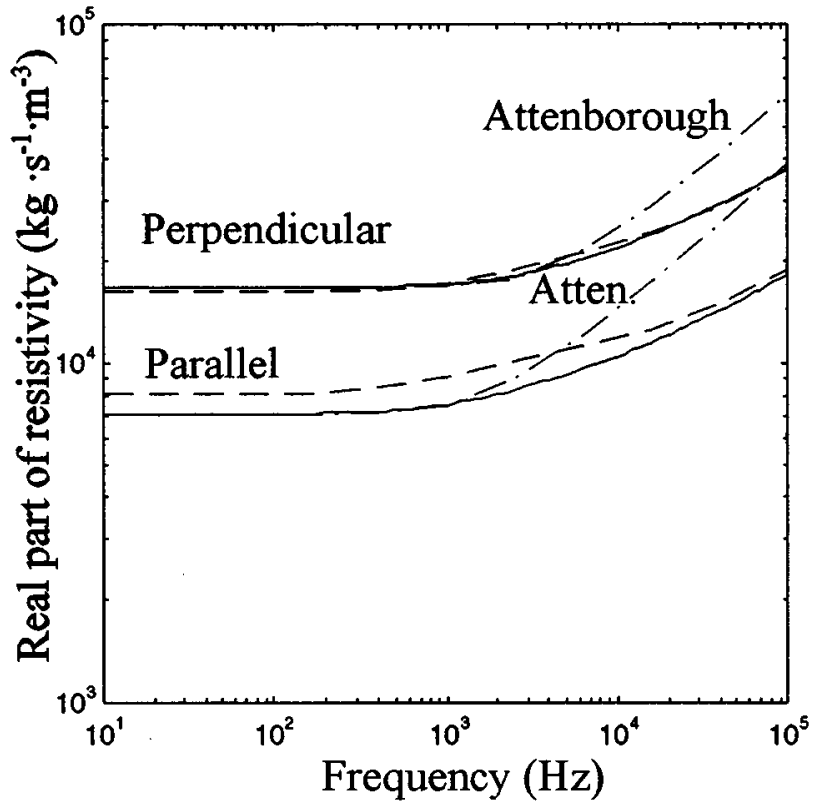

FIG. 2. The real part of the dynamic resistivity. The area per cylinder is $b^{2}$ with $b=76 \mu \mathrm{m}$, and the diameter of the cylinders is $6.8 \mu \mathrm{m}$. These numbers are valid for glass wool with a density of $16 \mathrm{~kg} / \mathrm{m}^{3}$. The lower dashed line is for flow parallel with cylinders calculated by the cell method. The lower full line is for parallel flow by the Brikman method. The upper dashed line is for flow perpendicular to cylinders by the cell method. The upper full line is for perpendicular flow by the Brinkman method. The dashed-dotted lines are found by the Attenborough approximation; the lower one is for parallel flow, and the upper one for perpendicular flow.
We divide both sides of the equation with the flux and rearrange the terms, and get the resistivity $R_{\|}$from $R_{\|}$ $=-(\partial p / \partial z) /\left\langle u_{z}\right\rangle$ and $\left\langle u_{z}\right\rangle=\langle\Psi\rangle / b^{2}$, where $b^{2}$ is the mean area of the Voronoi cells. The result is

$$
R_{\|}=\Omega^{-1}\left[-i \omega \rho_{0}-\frac{\eta 2 \pi a}{\langle\Psi\rangle}\left\langle\left[\frac{\partial u_{z}}{\partial r}\right]_{r=a}\right\rangle\right]
$$

where the porosity $\Omega$ is

$$
\Omega=\frac{b^{2}-\pi a^{2}}{b^{2}} .
$$

The first term in the bracket of Eq. (15) is the resistivity one would find if there were no cylinders. The mean value of the flux through a unit cell is found from Eqs. (12) and (6); thus

$$
\langle\Psi\rangle=\int_{a}^{\infty} \Psi(\rho) p_{b}\left(\pi \rho^{2}\right) 2 \pi \rho d \rho .
$$

The integral was calculated by numeric integration using Eqs. (12) and (6). By differentiation of Eq. (8) $\partial u_{z} / \partial r$ was found; then $\left[\partial u_{z} / \partial r\right]_{r=a}$ was substituted for $\Psi$ in Eq. (17), and $\left\langle\left[\partial u_{z} / \partial r\right]_{r=a}\right\rangle$ was found by numeric integration. The lower dashed line in Fig. 2 shows the real part of the resistivity, where the parameters used correspond to glass wool with mass density $16 \mathrm{~kg} / \mathrm{m}^{3}$, cylinder diameter $6.8 \mu \mathrm{m}$, and $b=76 \mu \mathrm{m}$. The volume density of fibers is 0.0063 . The lower dashed line in Fig. 3 shows the real part of the resistivity for cylinder diameter $6.8 \mu \mathrm{m}$, and $b=40 \mu \mathrm{m}$, corresponding to a volumen concentration of 0.040 .

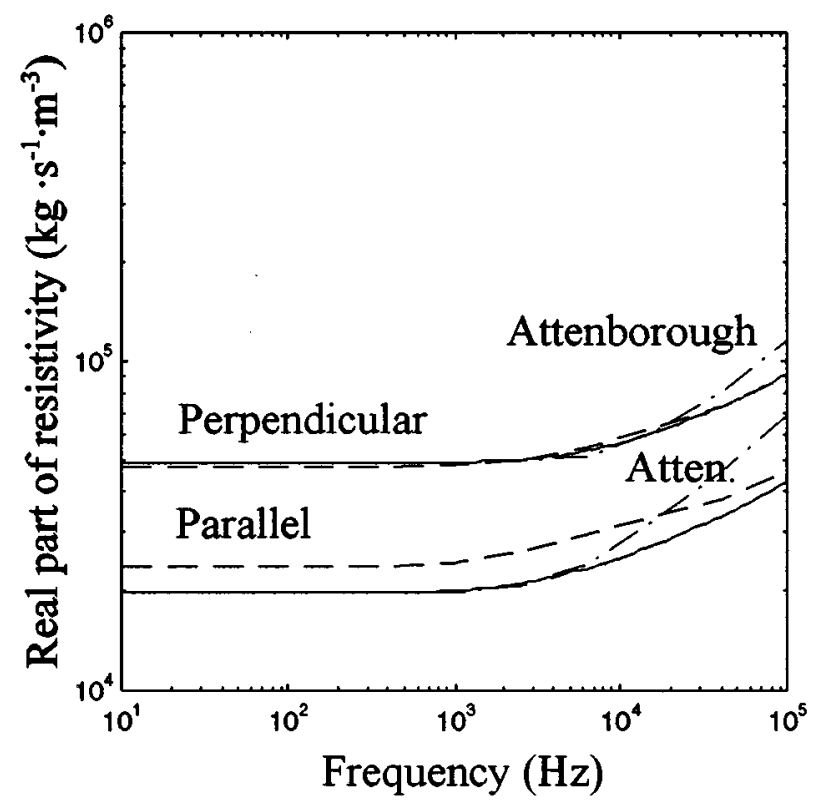

FIG. 3. The real part of the dynamic resistivity. The area per cylinder is $b^{2}$ with $b=50 \mu \mathrm{m}$, and the diameter of the cylinders is $6.8 \mu \mathrm{m}$. These numbers are valid for glass wool with a density of $40 \mathrm{~kg} / \mathrm{m}^{3}$. The lower dashed line is for flow parallel with cylinders calculated by the cell method. The lower full line is for parallel flow by the Brinkman method. The upper dashed line is for flow perpendicular to cylinders by the cell method. The upper full line is for perpendicular flow by the Brinkman method. The dashed-dotted lines are found by the Attenborough approximation; the lower one is for parallel flow, and the upper one for perpendicular flow. 


\section{B. Brinkman's self-consistent approximation}

A simple self-consistent (iterative) calculation of Brinkman has been used for many years to calculate the resistivity of dc flow. ${ }^{15}$ It can also be used to calculate the dynamic resistivity as will be shown in the following. Only one cylinder is considered, placed at the origin of a coordinate system; the influence of all the other cylinders on the one at the origin is taken care of by introducing a constant body force in the Navier-Stokes equation instead of the discrete forces from the cylinders. The force is proportional to the velocity, and the factor of proportionality is the "resistivity" $R_{\|}^{\prime}$. The equation for the velocity of the air becomes

$$
-i \omega \rho_{0} u_{z}=-\frac{\partial p}{\partial z}+\eta\left(\frac{\partial^{2} u_{z}}{\partial x^{2}}+\frac{\partial^{2} u_{z}}{\partial y^{2}}\right)-R_{\|}^{\prime} u_{z} .
$$

The force on a volume element is the gradient of the pressure; it equals the inertial forces plus the forces from the cylinders. That is, $-\partial p / \partial z=-i \omega \rho_{0} u_{z}+R_{\|}^{\prime} u_{z}$. From this and Eq. (1) follows $R_{\|}=R_{\|}^{\prime}-i \omega \rho_{0}$.

We regard the velocity as a function of the distance $r$ from the $z$ axis, and the boundary conditions are

$$
u_{z}(a)=0
$$

and

$$
u_{z}(\infty)=\langle u\rangle .
$$

A solution to Eq. (18) that fulfils these boundary conditions is

$$
u_{z}=\langle u\rangle\left[1-\frac{H_{0}^{1}\left(k_{\|}^{\prime} r\right)}{H_{0}^{1}\left(k_{\|}^{\prime} a\right)}\right],
$$

where

$$
k_{\|}^{\prime 2}=\left(i \omega \rho_{0}-R_{\|}^{\prime}\right) / \eta
$$

The force on one cylinder per length $F_{\|}$is

$$
F_{\|}=\left.2 \pi a \eta \frac{\partial u_{z}}{\partial r}\right|_{r=a} .
$$

We require that the distributed forces per volume equal the forces at the origin times the number of cylinders per volume. Thus

$$
R_{\|}^{\prime}\langle u\rangle=F_{\|} b^{2} .
$$

Set the velocity from Eq. (21) into (23) and use (24) to get

$$
R_{\|}^{\prime}=\frac{\eta 2 \pi k_{\|}^{\prime} a H_{1}^{1}\left(k_{\|}^{\prime} a\right)}{b^{2} H_{0}^{1}\left(k_{\|}^{\prime} a\right)} .
$$

Equations (22) and (25) are solved by an iterative process. First the value of $k_{\|}^{\prime}=i / b$ is chosen and set into (25); then a value of the resistivity $R_{\|}^{\prime}$ is computed. From the new value of the resistivity a new value of $k_{\|}^{\prime}$ is computed by (22), the new value of the wave vector is set into (25), and a new resistivity is computed, etc. After four iterations it was not possible to see any change on a graph of the resistivity as a function of the frequency. The calculation is "selfconsistent" when the resistivity is constant from one iteration to the next one.
The lower full line of Fig. 2 shows the real part of the resistivity computed for $a=3.4 \mu \mathrm{m}, b=76 \mu \mathrm{m}$. The lower full line of Fig. 3 shows the real part of the resistivity computed for $a=3.4 \mu \mathrm{m}, b=50 \mu \mathrm{m}$. The Brinkman method gives nearly the same result as the cell method; this has also been found by calculation of dc flow resistivity, ${ }^{15}$ which shows that the Brinkman approximation gives reasonably accurate values of the resistivity for volume concentration $<0.1$. The cell method tends to give too high a value of the resistivity because it was assumed that the cylinder was placed in the center of the Voronoi cells, which is not the case for a number of Voronoi cells, as one can see in Fig. 1. The Brinkman approximation is quite simple to implement on a computer, and accurate enough for the calculation of the resistivity of fiber materials.

\section{FLOW PERPENDICULAR TO CYLINDERS}

The flow is now perpendicular to the cylinders, which we assume are all parallel and have the same diameter, they are randomly distributed with constant density. We use a rectangular coordinate system with the $Z$ axis in the direction of the cylinder axes, and the $X$ axis in the direction of the flow. The Navier-Stokes equation (4) shall be solved, but in this case the solution is more complicated. The local air velocity $\mathbf{u}$ is expressed by the scalar potential $\phi$ and the vector potential A:

$$
\mathbf{u}=\boldsymbol{\nabla} \phi+\boldsymbol{\nabla} \times \mathbf{A} .
$$

Here the scalar potential must fulfil the Laplace equation

$$
\nabla^{2} \phi=0,
$$

because the flow is incompressible, Eq. (5).

An equation for the vector potential can be found by substitution of Eq. (26) into the Navier-Stokes equation (4),

$$
-\rho_{0} i \omega(\boldsymbol{\nabla} \phi+\nabla \times \mathbf{A})=\boldsymbol{\nabla} p+\eta \nabla^{2}(\boldsymbol{\nabla} \phi+\nabla \times \mathbf{A}) .
$$

This equation is fulfilled if

$$
-\rho_{0} i \omega \nabla \phi+\nabla p=0
$$

and

$$
-\rho_{0} i \omega \mathbf{A}+\eta \nabla^{2} \mathbf{A}=0 .
$$

We use a polar coordinate system with $r, \theta$ as coordinates, where

$$
x=r \cos \theta, \quad y=r \sin \theta .
$$

The scalar potential is a solution to Laplace's equation (27), and can be written as a Fourier series

$$
\begin{aligned}
\phi= & a_{0} \ln r+\sum_{n=1}^{\infty}\left[\left(a_{n} r+b_{n} r^{-n}\right) \cos n \theta\right. \\
& \left.+\left(c_{n} r+d_{n} r^{-n}\right) \sin n \theta\right] .
\end{aligned}
$$

Here $a_{n}, b_{n}, c_{n}, d_{n}$ are constant. The vector potential lies only in the $z$ direction because the flow is two dimensional. It is a solution to Eq. (30), and can be written as a Fourier series 


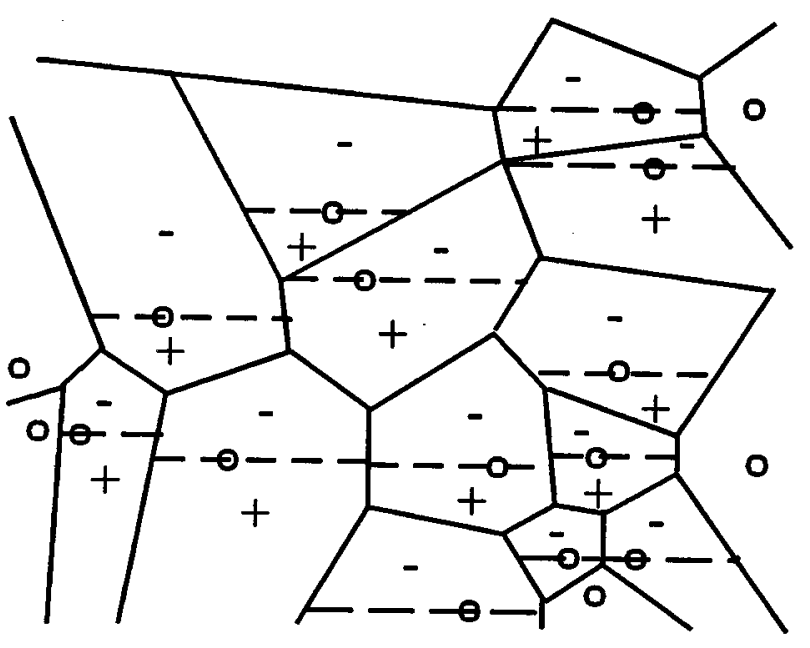

\section{Direction of flow}

FIG. 4. The rotation of the velocity in the cells. The dashed lines are parallel to the direction of the mean flow, which is from left to right. In the upper part of each cell the rotation is negative, in the lower part positive. The rotation is zero close to the border between the upper and lower part of neighbor cells.

$$
\begin{aligned}
A_{z}= & a_{0}^{\prime} H_{0}^{1}(k r)+b_{0}^{\prime} J_{0}(k r)+\sum_{n=1}^{\infty}\left[\left(a_{n}^{\prime} H_{n}^{1}(k r)+b_{n}^{\prime} J_{n}(k r)\right)\right. \\
& \left.\times \cos n \theta+\left(c_{n}^{\prime} H_{n}^{1}(k r)+d_{n}^{\prime} J_{n}(k r)\right) \sin n \theta\right],
\end{aligned}
$$

where $k$ is defined in Eq. (10), and $a_{n}^{\prime}, b_{n}^{\prime}, c_{n}^{\prime}, d_{n}^{\prime}$ are constants.

\section{A. The cell approximation}

By the flow between the cylinders of radius $a$ the rotation of the velocity vanishes close to the perimeter of each Voronoi cell. In Fig. 4 the flow is from left to right. The rotation $\partial u_{y} / \partial x-\partial u_{x} / \partial y$ is negative in the upper part of each cell and positive in the lower part. Where the upper part of one cell meets the lower part of another cell along the border, the rotation tends to be zero. This argument comes from a paper by Kuwabara ${ }^{16}$ on dc flow. The same assumption was used in Tarnow ${ }^{13}$ for calculation of the dc flow resistivity. It was shown that it gave reasonable results.

We use the cell approximation to compute the resistivity. We first consider a simple problem. One cylinder of a radius $a$ is given; this cylinder is surrounded coaxially with a cylinder surface of a radius $\rho$. The velocity is symmetric about $\theta=0$. Therefore, in the scalar potential of Eq. (32), only cosine terms are needed, and due to the circular geometry only the coefficient $a_{1}$ and $b_{1}$ are different from zero.

The rotation of the velocity is zero for $r=\rho$. This is an approximation to the boundary condition along the perimeter of each Voronoi cell. It will be shown that the rotation of the velocity is

$$
\boldsymbol{\nabla} \times \mathbf{u}=\boldsymbol{\nabla} \times \boldsymbol{\nabla} \times \mathbf{A}=-\left(\rho_{0} i \omega / \eta\right) \mathbf{A} .
$$

The vector potential is in the direction of the $Z$ axis perpendicular to the flow and does not depend on the $z$ coordinate because the flow is two dimensional. Therefore $\boldsymbol{\nabla} \cdot \mathbf{A}=0$, and the vector identity $\boldsymbol{\nabla} \times \boldsymbol{\nabla} \times \mathbf{A}=\boldsymbol{\nabla}(\boldsymbol{\nabla} \cdot \mathbf{A})-\nabla^{2} \mathbf{A}$ gives $\boldsymbol{\nabla} \times \boldsymbol{\nabla} \times \mathbf{A}=-\nabla^{2} \mathbf{A}$. Equation (34) then follows from Eq. (30). From Eq. (34) one sees that the vanishing of the rotation of the velocity implies that the vector potential must be zero. On the cylinder surface with radius $\rho$ we require that the vector potential vanish.

We first solve the Navier-Stokes equation in the circular cell. In order to make the formulas a little more simple, we use the Bessel function of Eq. (9) $Z_{i}(k r)$, which for $i=1$ is zero at the cylinder surface with radius $\rho$.

The velocity is symmetric about $\theta=0$, and the velocity equals the rotation of the vector potential; therefore, only the sine terms are kept in Eq. (33). In the circular cell only the first sine term is needed. Thus the vector potential can be written

$$
A_{z}=g Z_{1}(k r) \sin \theta,
$$

where $g$ is an integration constant.

The velocity in the direction of the radius $u_{r}$ is from Eqs. (26), (32), (35):

$$
u_{r}=\left(a_{1}-\frac{b_{1}}{r^{2}}+\frac{1}{r} g Z_{1}(k r)\right) \cos \theta,
$$

and the velocity perpendicular to the radius $u_{\theta}$ is

$$
u_{\theta}=-\left(a_{1}+\frac{b_{1}}{r^{2}}+g k Z_{1}^{\prime}(k r)\right) \sin \theta .
$$

Here the prime on the Bessel function indicates the derivative. The velocity must be zero at the internal cylinder surface, $r=a$; from this and the above formulas one gets

$$
a_{1}-\frac{b_{1}}{a^{2}}+\frac{1}{a} g Z_{1}(k a)=0
$$

and

$$
a_{1}+\frac{b_{1}}{a^{2}}+g k Z_{1}^{\prime}(k a)=0 .
$$

The pressure follows from Eqs. (29) and (32):

$$
p=i \omega \rho_{0}\left[a_{1} r+\frac{b_{1}}{r}\right] \cos \theta .
$$

For the random medium, we assume that the mean pressure gradient $\langle\nabla p\rangle$ is equal to the pressure drop over the cell divided by the cell diameter $2 \rho$, and get

$$
a_{1}+\frac{b_{1}}{\rho^{2}}=\frac{\langle\nabla p\rangle}{i \omega \rho_{0}} .
$$

Equations (38), (39), and (41) are three linear equations in the three unknowns $a_{1}, b_{1}, g$, when $\langle\nabla p\rangle$ is known. When they are solved, the velocity and pressure in the cell are known.

The resistivity will be computed by considering the energy flow. In general it is more accurate to use energy meth- 
ods to compute resistivity instead of using the definition Eq. (1) directly. The complex power $P$ of the medium in the volume $V$ is

$$
P=-\langle\nabla p\rangle^{*} \cdot\langle\mathbf{u}\rangle V,
$$

where ${ }^{*}$ means complex conjugation. The real part of this is the heat generated per volume. From the definition of resistivity Eq. (1) one gets $-\langle\nabla p\rangle=R_{\perp}\langle\mathbf{u}\rangle$, where $R_{\perp}$ is the resistivity for perpendicular flow. From this and Eq. (42) one gets

$$
R_{\perp}=\frac{|\langle\nabla p\rangle|^{2}}{P / V} .
$$

The power per length $P(\rho)$ inside the cylinder with radius $\rho$ can be calculated from

$$
P(\rho)=-\oint_{S} p^{*} \mathbf{u} \cdot d \mathbf{A}
$$

where the integration is done over the cylinder surface with radius $\rho$. After integration and use of (9) one gets

$$
P(\rho)=\frac{\left(\rho^{2} / a^{2}-1\right) Z_{0}(k a)+(2 / k a) Z_{1}(k a)}{\left(\rho^{2} / a^{2}+1\right) Z_{0}(k a)-(2 / k a) Z_{1}(k a)} \pi \rho^{2} \frac{|\nabla p|^{2}}{i \omega \rho_{0}} .
$$

The resistivity is calculated from

$$
R_{\perp}=\frac{|\nabla p|^{2} b^{2}}{\langle P\rangle}
$$

where the angular brackets mean an average value computed by the probability density in Eq. (6) in the following way:

$$
\langle P\rangle=\int_{a}^{\infty} P(\rho) p_{b}\left(\pi \rho^{2}\right) 2 \pi \rho d \rho .
$$

Here $P(\rho)$ is the function defined in (45), and $\bar{S}=b^{2}$ in (6).

\section{B. Brinkman's self-consistent approximation}

The dynamic resistivity for flow perpendicular to cylinders can be calculated by a self-consistent procedure similar to the one used for parallel flow. To the Navier-Stokes equation is added a body force $-R_{\perp}^{\prime} \mathbf{u}$ that equals the force from the cylinders per volume, where $R_{\perp}^{\prime}$ is the resistivity due to the forces on the cylinders. The Navier-Stokes equation becomes

$$
-i \omega \rho_{0} \mathbf{u}=-\nabla p+\eta \nabla^{2} \mathbf{u}-R_{\perp}^{\prime} \mathbf{u} .
$$

Only one cylinder at the origin of the coordinate system is considered. On the cylinder surface the velocity must be zero,

$$
\mathbf{u}(a)=0 ;
$$

and infinitely far from the cylinder at the origin the velocity equals the mean velocity $\langle u\rangle$, which is in the $X$ direction,

$$
\mathbf{u}(\infty)=(\langle u\rangle, 0,0) \text {. }
$$

A solution to Eq. (48) that fulfils the boundary conditions (49) and (50) can be written in the same way as Eqs. (36) and (37), but the special Bessel function $Z_{1}(k r)$ is replaced by a
Hankel function, which goes to zero as the radius goes to infinity. One gets

$$
u_{r}=\left(\langle u\rangle-\frac{b_{1}}{r^{2}}+\frac{1}{r} h H_{1}^{1}\left(k_{\perp}^{\prime} r\right)\right) \cos \theta
$$

and

$$
u_{\theta}=-\left(\langle u\rangle+\frac{b_{1}}{r^{2}}+h k_{\perp}^{\prime} H_{1}^{1^{\prime}}\left(k_{\perp}^{\prime} r\right)\right) \sin \theta .
$$

The prime on the Hankel function indicates the derivative, and $h$ is an integration constant. In Eqs. (50) and (51), $k_{\perp}^{\prime}$ is given by the right-hand side of Eq. (22) with $R_{\perp}^{\prime}$ instead of $R_{\|}^{\prime}$. The boundary condition Eq. (49), and Eqs. (51) and (52) give by use of the identity $H_{1}^{1^{\prime}}(w)=H_{0}^{1}(w)-(1 / w) H_{1}^{1}(w)$,

$$
h=-\frac{2\langle u\rangle}{k_{\perp}^{\prime} H_{0}^{1}\left(k_{\perp}^{\prime} a\right) .}
$$

From Eqs. (48), (49), (51), and (52) one finds

$$
b_{1}=a^{2}\left(1-\frac{2 H_{1}^{1}\left(k_{\perp}^{\prime} a\right)}{k_{\perp}^{\prime} a H_{0}^{1}\left(k_{\perp}^{\prime} a\right)}\right)\langle u\rangle .
$$

The force on the cylinder at the origin of the coordinate system may now be found from the pressure and the stress tensor. The calculation is similar to the calculation in section 20 of Landau and Lifschitz, ${ }^{11}$ where the Stokes formula for the viscous drag on a sphere is computed. There one finds the force written as an integral over the surface of the sphere; and this can be adapted to an integral over a cylinder, which is what is needed here. Section 15 of Landau and Lifschitz ${ }^{11}$ also gives the stress tensor in polar coordinates. One finds the force $F_{\perp}$ per length of the cylinder

$$
F_{\perp}=2 \pi \eta k_{\perp}^{\prime} a\left(-k_{\perp}^{\prime} a+\frac{2 H_{1}^{1}\left(k_{\perp}^{\prime} a\right)}{H_{0}^{1}\left(k_{\perp}^{\prime} a\right)}\right) .
$$

We require that the body force per volume equals the force from the cylinders per volume similar to Eq. (24), and get the resistivity

$$
R_{\perp}^{\prime}=\frac{2 \pi \eta k_{\perp}^{\prime} a}{b^{2}}\left(-k_{\perp}^{\prime} a+\frac{2 H_{1}^{1}\left(k_{\perp}^{\prime} a\right)}{H_{0}^{1}\left(k_{\perp}^{\prime} a\right)}\right) .
$$

We proceed in the same way for the case of parallel flow by iteration. We first choose a value of the wave vector, for example $k_{\perp}^{\prime}=i / b$, and calculate a resistivity from (56), then a new wave vector is calculated by Eq. (22), etc. until a stable value of the resistivity is found, which takes about four iterations. The inertia of the air between the cylinders is not included in the resistivity Eq. (56). Therefore the resistivity defined in Eq. (1) is

$$
R_{\perp}=-i \omega \rho_{0}+R_{\perp}^{\prime} .
$$

The upper full line of Fig. 2 shows the real part of the resistivity computed for $a=3.4 \mu \mathrm{m}, b=76 \mu \mathrm{m}$. The upper full line of Fig. 3 shows the real part of the resistivity computed for $a=3.4 \mu \mathrm{m}, b=50 \mu \mathrm{m}$. The Brinkman method gives nearly the same result as the cell method; this has also been found by calculation of dc flow resistivity, ${ }^{15}$ which shows that the Brinkman approximation gives reasonably accurate 


\section{DISCUSSION}

The assumption of parallel fibers is valid for glass wool plates produced by Glasuld, Scan-Gobain Denmark, which have fibers that are mainly parallel with the largest surface and with one edge, according to information from the producer. The measurements reported in Tarnow ${ }^{2}$ were made with sound propagation perpendicular to the largest surface of the plates. In this case one can assume that the sound velocity is perpendicular to the fibers. For other materials and uses one must make and average of the resistance tensor, but this depends on information about the orientation of the fibers in a given material and the direction of sound propagation. This information is not available. But I hope to make new measurements that can give more information on this. Anisotropy of glass wool has been measured by Allard et al. ${ }^{17}$ They found that the dc flow resistivity perpendicular to the fibers was two times the resistivity parallel with the fibers. This is in accordance with the low-frequency value found in the present paper and the values calculated in Tarnow. ${ }^{13}$ One can show that in the low-frequency limit, $R_{\perp}^{\prime} \approx 2 R_{\|}^{\prime}$ follows from Eqs. (25) and (56).

The resistivity has been compared with the model of Attenborough. Because the concentration of fibers is small, one must assume that the porosity and the tortuosity is 1 . The pore shape ratio must be 0.5 in order to obtain the proper low-frequency limit. We follow the definitions used by Stinson and Champoux. ${ }^{9}$ The low-frequency resistivity was calculated by the Brinkman method of the present paper and this was used as the dc resistivity $\sigma$. The Attenborough formula for the dynamic effective mass density becomes in this case

$$
\rho(\omega)=\frac{\rho_{0}}{1-2 J_{1}(w) / w J_{0}(w)},
$$

with $w=\sqrt{8 \rho_{0} i \omega / \sigma}$. The result of the calculation is shown in Figs. 2 and 3 as the dashed-dotted line. In Fig. 2 there are differences between the Brinkman approximation and the Attenborough formula at high audio frequencies. The Attenborough model assumes a circular tubes, but tubes with other cross-section shapes will not give very different highfrequency dependence because tubes have greater surfaces than fibers.

The imaginary part of the resistivity is approximately proportional to the frequency. In order to show graphically the difference between the different methods of calculating, the real part of the dynamic effective mass density is given. This is related to the imaginary part of the resistivity by $\operatorname{Im}\{R\}=-\omega \cdot \operatorname{Re}\{\rho(\omega)\} \quad$ which follows from $\rho(\omega)$ $=-i \omega R(\omega)$. Figure 5 shows $\operatorname{Re}\{\rho(\omega)\} / \rho_{0}$ for $a=3.4 \mu \mathrm{m}$, $b=76 \mu \mathrm{m}$. In Fig. 5 the full line is for flow parallel with cylinders (fibers) calculated by the Brinkman method Eqs. (22) and (25) and $R_{\|}=R_{\|}^{\prime}-i \omega \rho_{0}$. The dashed line is for parallel flow calculated by the Attenborough formula Eq. (58). The dotted line is for perpendicular flow; it was calculated by Brinkman's method Eqs. (22) and (56) and $R_{\perp}$ $=R_{\perp}^{\prime}-i \omega \rho_{0}$. The dashed-dotted line is for perpendicular

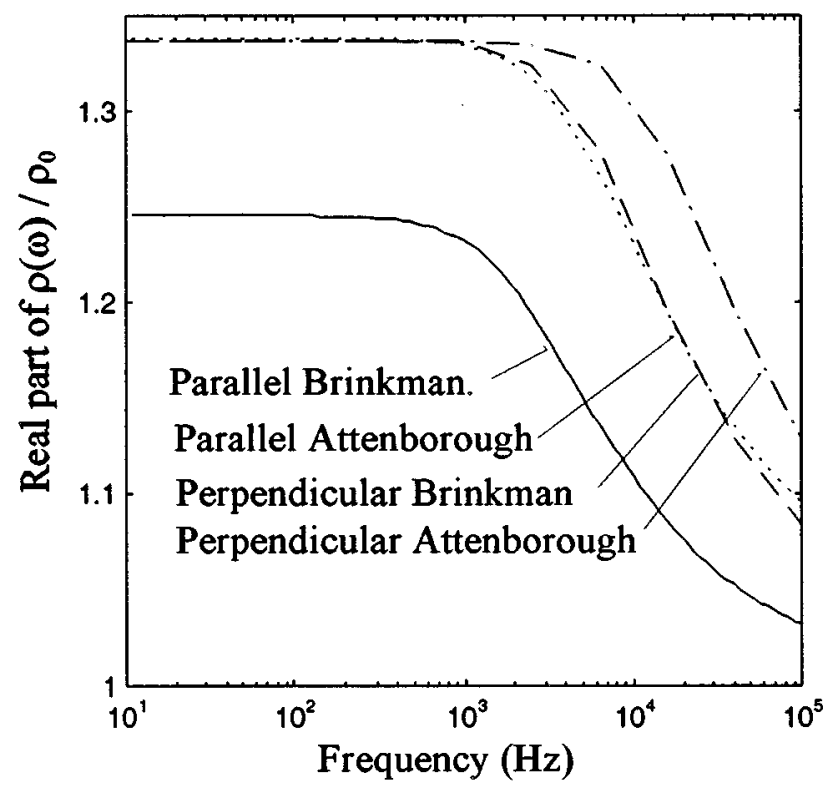

FIG. 5. The real part of the effective mass density. This is related to the imaginary part of the resistivity by $\operatorname{Im}\{R\}=-\omega \operatorname{Re}\{\rho(\omega)\}$. The figure shows $\operatorname{Re}\{\rho(\omega)\} / \rho_{0}$, where $\rho_{0}$ is the mass density of air. The area per cylinder is $b^{2}$ with $b=76 \mu \mathrm{m}$, and the diameter of the cylinders is $6.8 \mu \mathrm{m}$ corresponding to a mass density of glass wool of $16 \mathrm{~kg} / \mathrm{m}^{3}$. The full line is for flow parallel with cylinders (fibers) calculated by the Brinkman method. The dashed line is for parallel flow; it was calculated by the Attenborough formula Eq. (58). The dotted line is for perpendicular flow; it was calculated by Brinkman's method. The dashed-dotted line is for perpendicular flow; it was calculated by Attenborough's Eq. (58).

flow by Attenborough's Eq. (58). It is obvious that there are differences between the results of the different methods of calculation.

\section{CONCLUSION}

The dynamic flow resistivity tensor of fiber materials has been calculated for a model that consists of parallel cylinders of equal diameter. The resistivity was calculated from the density of cylinders and their diameter. Two cases were considered: flow parallel with and flow perpendicular to cylinders. From this and the symmetry of the resistivity tensor all the elements of the tensor are known.

The calculations were done with two approximations: a cell approximation and Brinkman's approximation. They gave results close to each other, but the Brinkman procedure is simpler to implement on a personal computer; it requires only a few lines of program in a high level mathematical program such as MATLAB. The accuracy of Brinkman's procedure is estimated to be of the order of $10 \%$ for fiber densities normally used in materials for acoustics; this was based on the calculations in this paper and Tarnow ${ }^{13}$ and Sangani and Yao. ${ }^{15}$

\footnotetext{
${ }^{1} \mathrm{~V}$. Tarnow, "Compressibility of air in fibrous materials," J. Acoust. Soc. Am. 99, 3010-3017 (1996).

${ }^{2} \mathrm{~V}$. Tarnow, "Measurement of sound propagation in glass wool," $\mathrm{J}$. Acoust. Soc. Am. 97, 2272-2281 (1995).

${ }^{3}$ C. Zwikker and C. W. Kosten, Sound Absorbing Materials (Elsevier, Amsterdam, 1949).

${ }^{4}$ M. A. Biot, "Theory of propagation of elastic waves in a fluid-saturated
} 
porous solid. II. Higher frequency range,' J. Acoust. Soc. Am. 28, 179191 (1956).

${ }^{5}$ R. F. Lambert, "The acoustical structure of highly porous open-cell foam," J. Acoust. Soc. Am. 72, 879-887 (1982).

${ }^{6}$ R. F. Lambert and J. S. Tesar, "Acoustic structure and propagation in highly porous, layered, fibrous materials,'” J. Acoust. Soc. Am. 76, 12311237 (1984).

${ }^{7} \mathrm{~K}$. Attenborough, "Acoustical characteristics of rigid fibrous adsorbents," J. Acoust. Soc. Am. 73, 785-799 (1983).

${ }^{8}$ M. R. Stinson, "The propagation of plane sound waves in narrow and wide circular tubes, and generalization to uniform tubes of arbitrary crosssectional shape,"' J. Acoust. Soc. Am. 89, 550-558 (1991).

${ }^{9}$ M. R. Stinson and Y. Champoux, "Propagation of sound and the assignment of shape factors in models of porous materials having simple pore geometries,'” J. Acoust. Soc. Am. 91, 685-695 (1992).

${ }^{10}$ J.-F. Allard and Y. Champoux, "New empirical equations for sound propagation in rigid frame fibrous materials," J. Acoust. Soc. Am. 91, 3346-3353 (1992).
${ }^{11}$ L. D. Landau and E. M. Lifschitz, Fluid Mechanics (Pergamon, Oxford, 1996).

${ }^{12}$ A. D. Pierce, Acoustics (Acoustical Society of America, New York, 1989), p. 513.

${ }^{13} \mathrm{~V}$. Tarnow, “Air flow resistivity of fibrous acoustic materials," J. Acoust. Soc. Am. 100, 3706-3713 (1996).

${ }^{14} \mathrm{P}$. N. Andrade and M. A. Fortes, "Distribution of cell volumes in a Voronoi partition,” Philos. Mag. B 58, 671-674 (1988).

${ }^{15}$ A. S. Sangani and C. Yao, "Transport processes in random arrays of cylinders. II. Viscous flow,” Phys. Fluids 31, 2435-2444 (1988).

${ }^{16} \mathrm{~S}$. Kuwabara, "The forces experienced by randomly distributed parallel circular cylinders or spheres in a viscous flow at small Reynolds numbers,"' J. Phys. Soc. Jpn. 14, 527-532 (1959).

${ }^{17}$ J.-F. Allard, R. Boudier, and A. l'Esperance, " Anisotropy effect in glass wool on normal impedance in oblique incidence," J. Sound Vib. 114, 233-238 (1987) 